\title{
Correction to: Pro-arrhythmogenic Effects of the V141M KCNQ1 Mutation in Short QT Syndrome and Its Potential Therapeutic Targets: Insights from Modeling
}

\author{
Hsiang-Chun Lee ${ }^{1,2,3,4,5,6} \cdot$ Yoram Rudy ${ }^{1} \cdot$ Hongwu Liang ${ }^{1} \cdot$ Chih-Chieh Chen ${ }^{6}$. Ching-Hsing Luo ${ }^{7}$. \\ Sheng-Hsiung Sheu ${ }^{2,3,4} \cdot$ Jianmin $\mathrm{Cui}^{1}$
}

Published online: 9 February 2018

(c) Taiwanese Society of Biomedical Engineering 2018

\section{Correction to: J. Med. Biol. Eng. (2017) 37:780-789 https://doi.org/10.1007/s40846-017-0257-x}

The article "Pro-arrhythmogenic Effects of the V141M KCNQ1 Mutation in Short QT Syndrome and Its Potential Therapeutic Targets: Insights from Modeling", written by Hsiang-Chun Lee, Yoram Rudy, Hongwu Liang, Chih-Chieh Chen, Ching-Hsing Luo, Sheng-Hsiung Sheu, Jianmin Cui was originally published Online First without open access. After publication in volume [37], issue [5], page [780-789] the author decided to opt for Open Choice and to make the article an open access publication. Therefore, the copyright of the article has been changed to (C) The Author(s) [2018] and the article is forthwith distributed under the terms of the Creative Commons Attribution 4.0 International License (http://creativecommons.org/licenses/by/4.0/), which permits use, duplication, adaptation, distribution and reproduction in any medium or format, as long as you give appropriate credit to the original author(s) and the source, provide a link to the Creative Commons license, and indicate if changes were made.

The original article can be found online at https://doi.org/10.1007/ s40846-017-0257-x.

Sheng-Hsiung Sheu

sheush@kmu.edu.tw

$\triangle$ Jianmin Cui

jcui@wustl.edu

1 Cardiac Bioelectricity and Arrhythmia Center, Washington

University in St. Louis, St. Louis, MO 63130, USA

2 Division of Cardiology, Department of Internal Medicine, Kaohsiung Medical University Hospital, Kaohsiung Medical University, 100 Tzyou 1st Rd, Kaohsiung 807, Taiwan

3 Faculty of Medicine, College of Medicine, Kaohsiung Medical University, Kaohsiung 807, Taiwan

4 Center for Lipid Biosciences, Kaohsiung Medical University Hospital, Kaohsiung Medical University, Kaohsiung 807, Taiwan

5 Lipid Science and Aging Research Center, Kaohsiung Medical University, Kaohsiung 807, Taiwan

6 Institute of Medical Science and Technology, National Sun Yat-sen University, Kaohsiung 804, Taiwan

7 Department of Electric Engineering, National Cheng Kung University, Tainan 804 , Taiwan 